VHDL: HARDWARE DESCRIPTION AND DESIGN 


\title{
VHDL: HARDWARE DESCRIPTION AND DESIGN
}

by

\author{
ROGER LIPSETT \\ CARL F. SCHAEFER \\ CARY USSERY
}

Intermetrics, Inc.

KLUWER ACADEMIC PUBLISHERS

Boston/Dordrecht/London 
Distributors for North America:

Kluwer Academic Publishers

101 Philip Drive

Assinippi Park

Norwell, Massachusetts 02061 USA

Distributors for all other countries:

Kluwer Academic Publishers Group

Distribution Centre

Post Office Box 322

3300 AH Dordrecht, THE NETHERLANDS

Consulting Editor: Jonathan Allen, Massachusetts Institute of Technology

\section{Library of Congress Cataloging-in-Publication Data}

Lipsett, Roger, 1950-

VHDL: Hardware description and design.

Bibliography: p.

Includes index.

1. Computer input-output equipment-Computer

simulation. 2. VHDL (Computer program language)
I. Schaefer, Carl F., 1945-
II. Ussery, Cary,

1962- III. Title.

TK7887.5.L57 $1989 \quad 621.39 ' 2 \quad 89-11114$

ISBN-13: 978-1-4612-8901-2

e-ISBN-13: 978-1-4613-1631-2

DOI: $10.1007 / 978-1-4613-1631-2$

Copyright (ㄷ 1989 by Kluwer Academic Publishers

Softcover reprint of the hardcover 1st edition 1989

Fifteenth Printing 1998

All rights reserved. No part of this publication may be reproduced, stored in a retrieval system or transmitted in any form or by any means, mechanical, photocopying, recording, or otherwise, without the prior written permission of the publisher, Kluwer Academic Publishers, 101 Philip Drive, Assinippi Park, Norwell, Massachusetts 02061. 


\section{Table of Contents}

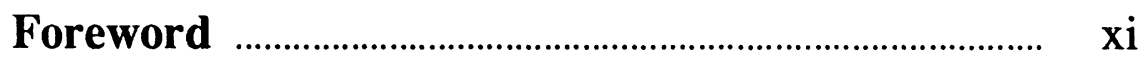

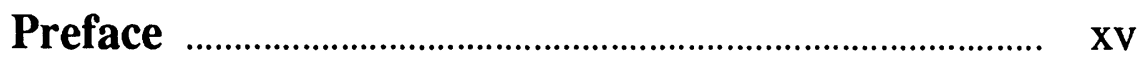

Overview of the Book ............................................................. xvi

Acknowledgements ..................................................................... xvii

Chapter 1 - Introduction ……………………………….... 1

Why VHDL ......................................................................... 2

Terminology and Conventions ................................................. 5

Chapter 2 - A Model of Hardware .............................. 7

A Model of Behavior ................................................................ 8

A Model of Time ………………………………………..... 11

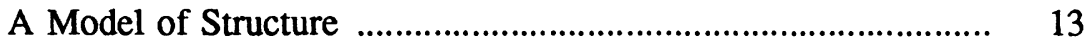

Chapter 3 - Basics ............................................................... 17

Structure and Behavior ......................................................... 18

Data Types and Objects ......................................................... 23

Data Types ...................................................................... 23

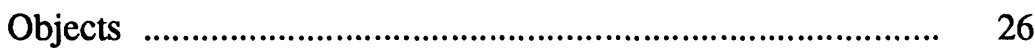

Hooking Constructs Together ….............................................. 27

Interface Lists ................................................................. 28

Association Lists ............................................................. 29

Major VHDL Constructs ……................................................. 30

Entity Declarations ............................................................. 31

Architecture Bodies .............................................................. 33

Subprograms ................................................................. 34

Packages and Use Clauses …………………….................. $\quad 36$

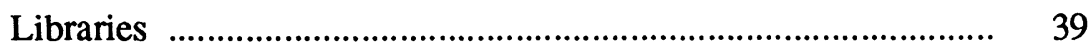

Library Units and Order of Analysis ……............................ 39

Visibility of a Primary Unit and Libraries ............................. 40 
Chapter 4 - Data Types .................................................... 45

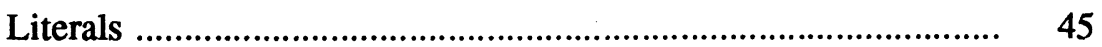

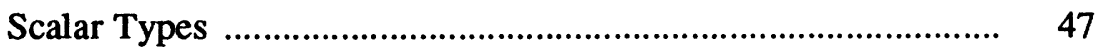

Composite Types ..................................................................... 49

Aggregates and String Literals …......................................... 51

Referencing Elements of Composites .................................... 54

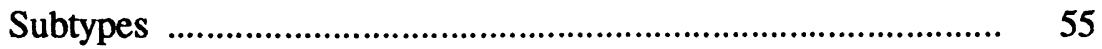

Attributes ..................................................................... 58

Predefined Operators .......................................................... 61

Chapter 5 - Behavioral Description .............................. 65

Process Statements ............................................................... 65

The Wait Statement: Activation and Suspension .................. $\quad 66$

Behavioral Modeling - Sequential View ................................... 68

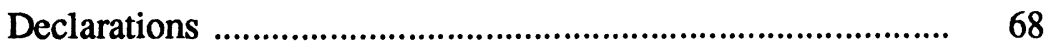

Sequential Assignment ...................................................... 69

Signal Assignment ....................................................... 70

Signal Drivers ............................................................. 73

Delay in Signal Assignments ......................................... 75

Variable Assignment .................................................. 82

Sequential Control ............................................................. 84

Conditional Control ...................................................... 84

Iterative Control ........................................................... 88

Other Sequential Statements ................................................ 92

The Assertion Statement …............................................. 92

Procedure Calls .............................................................. 94

The Return Statement ….................................................. 94

The Null Statement ..................................................... 95

Behavioral Modeling - Concurrent View ................................... 95

Concurrent Statements and Equivalent Processes ................ 96

Concurrent Signal Assignment ........................................ 96

Concurrent Assertion Statement ...................................... 101

Resolved Signals .............................................................. 103

A Counter Element ............................................................. 105

Chapter 6 - Structural Description ............................. 107

Basic Features of Structural Description .................................. 108 
Ports in Entity Declarations .................................................. 108

Port Modes and Direction of Data Flow ............................. 109

Ports in Component Declarations ...................................... 110

Component Instantiation Statements .................................... 110

Example: A Simple ALU ............................................... 114

Example: A Decoder ...................................................... 118

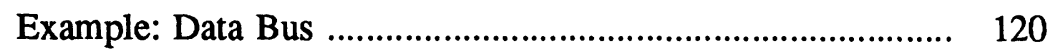

Regular Structures ............................................................. 124

Generate Statements ....................................................... 124

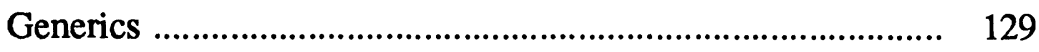

Configuration Specifications ................................................. 131

Default Values and Unconnected Ports .................................. 138

Default Values ................................................................ 138

Unconnected Ports ............................................................. 142

Chapter 7 - Large Scale Design .................................... 145

Managing Shared Designs ........................................................ 146

Design Libraries and their Implementation .......................... 146

Predefined Design Libraries .................................................. 147

The Use of Libraries for Revision Management ................... 148

Visibility and the Analysis Context .......................................... 149

Name Visibility in VHDL ............................................... 149

Access to External VHDL Libraries ................................... 153

Partitioning a Design ............................................................ 155

Concurrent and Sequential Procedure Calls ......................... 156

The Block Statement .......................................................... 158

Component Instantiations and Blocks................................ 159

Sharing Data Within a Design .................................................. 163

Specifying a Design Configuration ........................................... 167

How Component Binding Occurs ....................................... 168

Type Incompatibilities in Component Binding ...................... 173

Mixing Structure and Behavior ................................................ 175

Chapter 8 - A Complete Example …............................ 177

The Traffic Light Controller ........................................................ 178

Creating the Specification ........................................................ 179

Defining the System Types ................................................... 179 
Creating the Interface ........................................................ 180

The Body of the Specification ............................................. 181

Creating a Test Bench ...................................................... 186

Partitioning the Design ....................................................... 188

Choosing a Type Representation ......................................... 189

Revising the Specification ................................................. 193

The First Partition .......................................................... 195

The Second Partition ........................................................ 198

Starting the Implementation ................................................. 201

Setting Up the PLA ............................................................. 204

Chapter 9 - Advanced Features ................................. 209

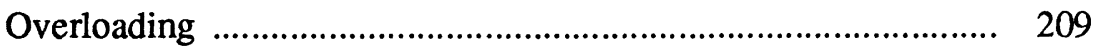

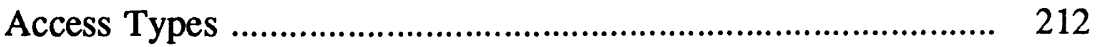

File Types and I/O ........................................................... 216

User-Defined Attributes ........................................................... 219

Signal-Related Attributes ...................................................... 221

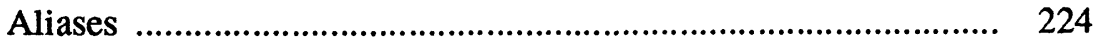

Association by Subelement ……............................................. 225

Guarded Assignment Statements .......................................... 227

Disconnection Specifications ............................................... 228

Null Transactions .............................................................. $\quad 230$

Chapter 10 - VHDL in Use ........................................ 233

A Device Controller .......................................................... 234

Setup and Hold Timing ....................................................... 240

A Neural Net ................................................................... 247

A Systolic Array Multiplier …………..................................... 253

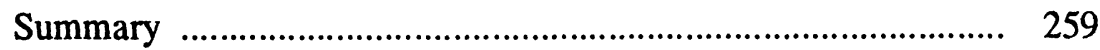

Appendix A - Predefined Environment ..................... 261

Reserved Words ...................................................................... 261

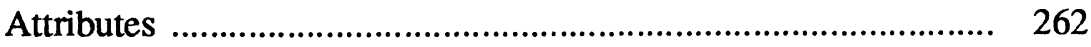

Type and Subtype Attributes .......................................... 262

Array Attributes ............................................................. 263

Signal-Valued Attributes ................................................... 264

Signal-Related Attributes .................................................... 265 


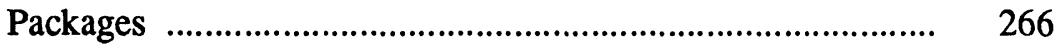

The Package STANDARD ............................................... 266

The Package TEXTIO ...................................................... 268

Appendix B - VHDL Syntax ........................... 273

Appendix C - Suggested Reading .................... 293

Index ......................................................... 295 


\section{Foreword}

VHDL is a comprehensive language that allows a user to deal with design complexity. Design, and the data representing a design, are complex by the very nature of a modern digital system constructed from VLSI chips. VHDL is the first language to allow one to capture all the nuances of that complexity, and to effectively manage the data and the design process. As this book shows, VHDL is not by its nature a complex language.

In 1980, the U.S. Government launched a very aggressive effort to advance the state-of-the-art in silicon technology. The objective was to significantly enhance operating performance and circuit density for Very Large Scale Integration (VLSI) silicon chips. The U.S. Government realized that in order for contractors to be able to work together to develop VLSI products, to document the resulting designs, to be able to reuse the designs in future products, and to efficiently upgrade existing designs, they needed a common communication medium for the design data. They wanted the design descriptions to be computer readable and executable. They also recognized that with the high densities envisioned for the U.S. Government's Very High Speed Integrated Circuit (VHSIC) chips and the large systems required in future procurements, a means of streamlining the design process and managing the large volumes of design data was required. Thus was born the concept of a standard hardware design and description language to solve all of these problems. 
In 1983, the U.S. Government issued a Request for Proposal to develop the language and to implement a set of tools for use with the language. The winner of the competitive bidding was a team composed of Intermetrics, International Business Machines, and Texas Instruments. As the lead engineer for the IBM part of the development effort, and as the chairman of the IEEE Computer Society Design Automation Standards Subcommittee, I saw directly the benefits that would accrue to industry from a standard hardware description language. VHDL is a descriptive language, one that is human and machine readable, so that it is well suited to preserve design specifications. VHDL is also a leading edge design language, one that may be used with many types of design tools such as simulators, design synthesizers, silicon compilers, placement and wiring tools, test generators, architectural specification and analysis tools, and timing analyzers, and can support research in all of these areas. At this time, many such tools are coming to the market place and university research in the named areas, which has been underway for years before the advent of VHDL, is proceeding to address itself to the use of VHDL. Why is this so?

As previously mentioned, a major power of VHDL is that it is a standard. Thus, industry can more easily communicate designs among participants in a design process. This ability to communicate designs is equally important in the research field, since, with VHDL, collaboration between researchers at various institutions becomes easier. But, standardization is not sufficient for the ground-swell of interest in the language. VHDL also has to be technically excellent and capable of allowing designers and researchers to describe the concepts they are developing and utilize the descriptions with tools in a way that simplifies the design or research process. VHDL achieves these goals.

The scope of VHDL covers the description of architectural description to gate level description. The language is hierarchical and mixed-level simulation is supported. The concepts embodied in the timing model for the language mirror real hardware -- the VHDL models of designs behave like real hardware. Many other excellent languages cover subsets of the capabilities that exist within VHDL, but none are as comprehensive. They do not cover the wide range covered by VHDL. Equally important, because VHDL is an IEEE standard, the language will have a significant effect on life-cycle support of products described in VHDL. At high levels of abstraction, the language makes an excellent specification medium for future designs to be created in new technologies or with alternative architectures. At lower levels of abstraction, the language serves well as a specification of what is to be fabricated.

All in all, VHDL is a "language for all seasons," one that supports design automation research, design and test, and product life-cycle. I take my hat off to the scores of people that have participated in the development of the VHDL concept and had the foresight to help bring it 
to fruition. I also salute the authors of this book for helping to bring understanding in the use of VHDL.

\author{
Ronald Waxman \\ University of Virginia \\ Charlottesville, Virginia \\ March 20, 1989
}




\section{Preface}

The VHSIC Hardware Description Language (VHDL) is a new hardware description language developed, starting in 1981, by the Very High Speed Integrated Circuits (VHSIC) Program Office of the Department of Defense for use as a standard language in the microelectronics community. This language represents a new step in the evolution of language support for hardware design. The recognized need for managing the complexity of information needed for digital design has driven the development of VHDL.

The acceptance process for a new language can be slow, especially for a language as rich in features as VHDL. The largest factor in this process is the dissemination of information about what the language is and, more importantly, how to use it. To date, little information has been available to the design community to explain what VHDL is, how it relates to what designers know, and how it can be integrated into the design process.

The authors of this book have, since the beginning of the VHDL program, been involved in developing the language, building tools that support the language, and using the language to build hardware descriptions. Intermetrics, Inc., for whom the authors work, was the Government's prime contractor for the original development of VHDL. Both Intermetrics and the authors are committed to the widespread use of VHDL as a hardware description language in the design community. This book represents an opportunity to inform; to share the knowledge 
of VHDL we have gained over the past five years.

This book describes the VHDL language and discusses ways in which VHDL can be used. It is intended to introduce the reader to all aspects of VHDL and to specific language constructs. It is not theoretical in nature and does not rigorously discuss the formal definition of the language; this approach is left to the Reference Manual for the language. What the book does do is take a look at the language feature by feature to provide an understanding of how each feature works and how it is integrated into the modeling process. This book is intended to give hardware designers and software model developers a thorough understanding of the VHDL language both as a language and as a design tool. We do not suggest particular ways of designing given hardware devices, but rather seek to provide the designer with an understanding of VHDL sufficient to allow him or her to best choose how to perform design.

The book is suitable for working professionals as well as graduate or advanced undergraduate study. Designers can view this book as a way to get acquainted with VHDL and as a reference for work with VHDL, while students and professors will find the book useful as a teaching tool for hardware design and hardware description languages in general, as well as VHDL in particular.

The presented material assumes a working knowledge of digital hardware design, as well as some familiarity with a high level programming language such as $\mathrm{C}$, Pascal, or Ada.

\section{Overview of the Book}

The book is divided into ten chapters.

The first chapter is an introductory chapter which gives a brief history of VHDL, examines reasons for using VHDL and discusses terminology and conventions used throughout the book. The second chapter introduces the model of digital devices on which VHDL is based. It discusses the main semantic content of the language without going into the syntax of the constructs used within the model.

Chapter 3 introduces some basic elements of VHDL. These elements will be used throughout the rest of the book. Chapter 4 examines data types in the language in detail. Some readers might want to skim this chapter at first and then return to it as the sophistication of their VHDL models evolves to require the inclusion of user-defined data types.

The next three chapters, Chapters 5, 6, and 7, discuss VHDL from three viewpoints. Chapter 5 discusses using VHDL to describe the behavior of a hardware device. Chapter 6 discusses using VHDL to describe the structure of a hardware device. Chapter 7 discusses 
combining these two viewpoints into a model of a large hardware device. All these views of modeling are supported by the language. These chapters introduce the reader to the bulk of the VHDL language from a functional perspective instead of from a language definition perspective.

Chapter 8 ties the concepts presented in the previous chapters into a single design example. The design is a traffic light controller which is developed from a high level specification into a PLA implementation.

Chapter 9 looks at some of the more advanced features of the language. These features are used for high level modeling, test vector input, and other specialized functions.

Chapter 10 works through a few design examples as a way of illustrating the use of VHDL in system modeling and abstract design. Other books which treat VHDL tend toward the detailed gate level or board level modeling techniques. Here we try to illustrate some of the higher level features of VHDL and its uses in abstract modeling.

\section{Acknowledgements}

Many people gave us support, advice, and commentary on the earlier drafts of this book. In particular, our peers at Intermetrics provided extensive assistance and criticism. Special thanks are due to $\mathrm{Al}$ Gilman, Doug Dunlop, Victor Berman, and, especially, Kathy McKinley. They were all subjected to questionable prose and countless technical errors which they handled gracefully, and with beneficial results.

We would like to express our appreciation for the support and resources provided by Intermetrics, Inc. This book was developed on three different computers in three different cities and would not have been possible without the cooperation of Intermetrics. We would especially like to thank Bill Carlson, Victor Berman and Bill Bail at Intermetrics for the direct support they have given this book.

We would like to thank Carl Harris at Kluwer Academic and Rachael Rusting at Intermetrics, whose patience and forbearance have helped to make this book a reality and to motivate the authors. Paula Gillis of Intermetrics is responsible for the cover art. Peter Connor contributed time and ideas during the early stages.

A number of people have provided valuable comments on drafts of this book. Dr. David Hemmendinger and John van Tassel of Wright State University, Capt. John Evans of the Air Force Foreign Technology Division, and Lt. Karen Serafino of the Electronic Technology Laboratory at the Wright Research and Development Center deserve special recognition for their careful review of the text and the analysis and simulation of all the examples. 
Over the past five years, many people have been involved with VHDL in one form or another. While we cannot mention all of these people here, we salute the effort and creativity of those who helped to bring VHDL to life. A few individuals deserve attention for keeping VHDL alive and well over the course of the past 5 years: Allen Dewey, who was the original Project Officer for the Air Force at Wright Field; Dr. John Hines, who has been a key factor in getting VHDL going and keeping the air in its sails; and Larry Saunders who, in addition to being a major proponent of VHDL in and out of IBM, chaired the IEEE VHDL Analysis and Standardization Group which carved out the current VHDL standard. 
VHDL: HARDWARE DESCRIPTION AND DESIGN 